\title{
Research on the Automatic Detection Method of Tunnel Clearance Based on Point Cloud Data
}

\author{
Jun HAN ${ }^{\mathrm{a}, \mathrm{b}, \mathrm{c}}$, Guodong $\mathrm{CHEN}^{\mathrm{d}}$, Tao LIU ${ }^{\mathrm{a}, \mathrm{b}, \mathrm{c}, 1}$, and Qian YANG ${ }^{\mathrm{d}}$ \\ ${ }^{a}$ Faculty of Geomatics, Lanzhou Jiaotong University, Lanzhou 730070 \\ ${ }^{b}$ National-Local Joint Engineering Research Center of Technologies and Applications \\ for National Geographic State Monitoring, Lanzhou 730070, China \\ ${ }^{c}$ Gansu Provincial Engineering Laboratory for National Geographic State Monitoring, \\ Lanzhou 730070, China \\ ${ }^{d}$ College of Civil Engineering, Lanzhou Jiaotong University, Lanzhou 730070, China
}

\begin{abstract}
Due to the deformation of the tunnel and the abnormal outburst of internal facilities, the existing railway tunnel line needs to be inspected regularly. However, the existing detection methods have some shortcomings, such as large measurement interference, low efficiency, discontinuity of section, and independence with the track structure. Therefore, an automatic detection method of tunnel space clearance based on point cloud data is proposed. By fitting the central axis of the tunnel, the extraction can be realized at any position of the tunnel. The coordinate system of tunnel gauge detection based on rail top surface is established, and different types of tunnel gauge frames are introduced. The improved ray algorithm method is used to realize automatic detection and analysis of various tunnel types. Field experiments on existing railway tunnels show that the method can accurately obtain the limit point and size of the tunnel. The cross-section of transgression is obtained. It can meet the requirements of tunnel detection accuracy and has great practicability in tunnel disease detection.
\end{abstract}

Keywords. Point cloud data, tunnel clearance, section extraction, improved ray method, automatic detection, judgment of invasion

\section{Introduction}

Tunnel gauge is a kind of railway structure approaching gauge, which is a contour close to the cross-section of the vehicle and vertical to the line plane. The construction gauge is a cross-sectional profile with a specific dimension, which is based on the rail surface, perpendicular to the railway center line in a horizontal straight line, and close to the locomotive and rolling stock gauge. To ensure the safety of train operation, according to relevant regulations, except for the equipment that must contact with the locomotive and the locomotive itself, the rest shall not intrude into this contour [1]. At present, there are many detection methods for railway tunnel clearance in China. The gauge car of the retractable frame can be made according to the gauge standard [2], but the frame of the detection vehicle needs to be adjusted manually in different road sections. The total station and reflection prism are used for single-point measurement [3]. However, due to

1 Corresponding Author, Tao Liu, professor, Lanzhou Jiaotong University, Lanzhou, China, ltaochina@foxmail.com 
heavy manual work, the obtained tunnel contour is separated from the track position, which can not accurately reflect the corresponding mutual position relationship between the two, resulting in a large error in gauge detection. According to the principle of CCD video camera, a system for tunnel clearance detection using video camera technology was developed [4]. However, the system has high requirements for light environments, and its application in the tunnel is limited. The laser tunnel profiler can directly obtain the tunnel section by using two-dimensional laser measurement [5], but only a single section perpendicular to the tunnel centerline can be detected at one time. The trolley loaded with laser ranging sensor is also introduced into the tunnel clearance detection [6-8], but the car is easy to be affected by inaccurate speed measurement and idling slip. France, Germany, and other countries have developed tunnel gauge detection vehicles based on multi-sensor integration [9,10], which can quickly and dynamically obtain tunnel cross-section, but it has great interference to traffic and high detection cost, so vibration compensation should be considered.

Ground 3D laser scanner can obtain 3D point cloud data of objects with high precision by non-contact measurement and has low requirements for the light environment [11-13]. In this paper, an integrated automatic detection method of rail and tunnel space gauge based on point cloud data is proposed. Through processing and analysis of 3D laser point cloud, the tunnel section is extracted, and different types of bounding boxes are introduced for calculation to realize the bound detection of railway tunnels. The overall technical process is shown in Figure 1.

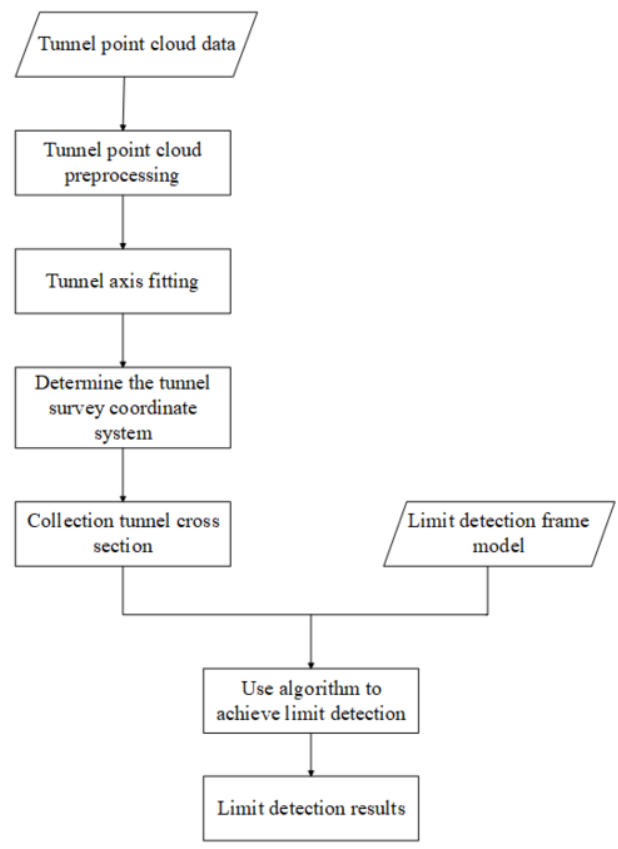

Figure 1. Flow chart of tunnel boundary detection technology 


\section{Point Cloud Acquisition and Preprocessing of Tunnel}

Considering the narrow and long tunnel structure, according to the ideas provided in reference [14], the method of setting stations at a certain distance in the center of the line is adopted for scanning, and the data of adjacent stations are spliced by three noncollinear target balls. However, due to the influence of dust, air pressure, and other external factors in the tunnel, the isolated noise points will inevitably be generated, which should be filtered out, that is to say, the point cloud should be denoised. The method is to construct a k-d tree [15], and then search for the number of near-infrared points within the radius of a certain point. If it is less than the predetermined threshold, the point is judged to be an isolated noise point. At the same time, this method can better maintain the original position of the point cloud.

\section{Extraction Method of Tunnel Cross Section}

In the detection of tunnel clearance, analyzing the cross-section of the tunnel is necessary, so it is necessary to extract the tunnel axis. At any point on the central axis, the direction of the tunnel at this point can be calculated, and the tunnel direction is orthogonal to the cross-section to be intercepted [16,17]. The amount of point cloud data after splicing and denoising is as many as several billion, so it is difficult to calculate directly. If the point cloud is diluted too much and the accuracy of axis extraction is affected, the segmentation processing method can be used to extract the tunnel axis to improve the operation efficiency.

\subsection{Tunnel Axis Fitting}

The 3D point cloud data is projected on the XOY surface, as shown in Figure 2. The minimum and maximum values in the $\mathrm{Y}$ direction are taken as the starting and ending points, and the reasonable step size $\Delta \mathrm{y}=0.05 \mathrm{M}$ is set along the $\mathrm{Y}$ direction. Then, the boundary points on both sides of the tunnel are extracted by the differential step method. The specific step of differential step method is to subdivide the point cloud into several thin strips of equal length along the y-axis, and then search for the points with the largest and smallest x-coordinate values on each element. As shown in Figure 3, the obtained point set 1 and point set 2 constitute the boundary points.

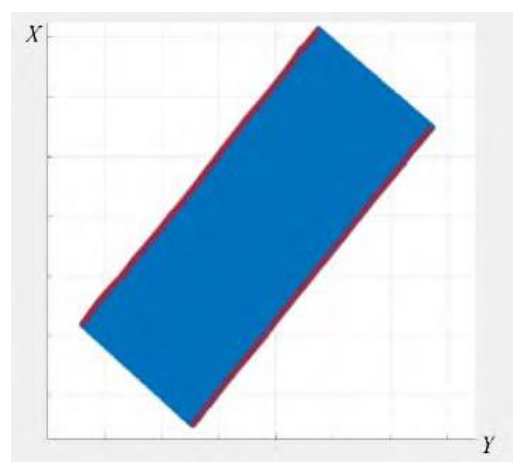

Figure 2. The Projection of point cloud on XOY plane. 


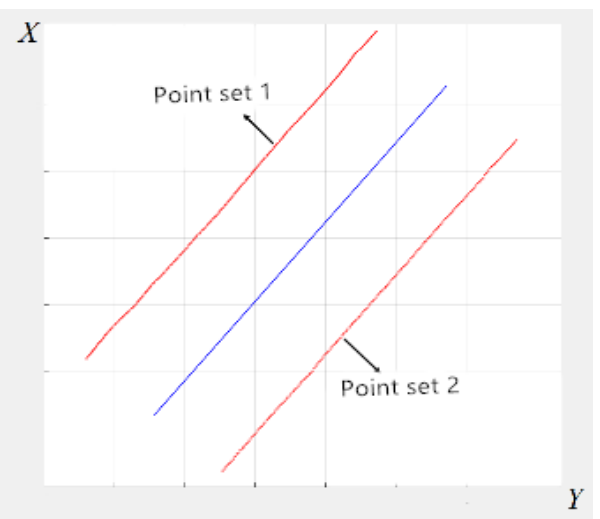

Figure 3. Fitting of boundary points and central axis.

Each point in point set 1 can find the nearest point in point set 2 , which constitutes a pair of boundary points. The midpoint of each pair of boundary points is calculated, and the central axis is fitted with conic according to a series of midpoint coordinates. In the same way, the point cloud is projected on the YOZ plane, and the central axis is fitted. Finally, the central axis of the tunnel can be expressed as the equation of $\mathrm{X}, \mathrm{Z}$ about $\mathrm{Y}$

$$
\left\{\begin{array}{l}
x=a_{0}+a_{1} y+a_{2} y^{2} \\
\mathrm{z}=b_{0}+b_{1} y+b_{2} y^{2}
\end{array}\right.
$$

\subsection{Tunnel Section Interception.}

The tunnel section is a plane perpendicular to the tunnel axis at all times. To intercept the cross-section in the orthogonal direction of the tunnel direction, the direction of the tunnel is determined according to the tunnel axis, and then the matrix basis is adjusted by rotating the corresponding angle so that the point cloud of the section at any position of the tunnel axis can be extracted. The continuous extraction of tunnel sections can be carried out by selecting the position of starting and ending points on the tunnel axis and preset the section interval. The normal vector of any point $\mathrm{P}$ on the central axis is $v_{1}\left(\begin{array}{lll}1 & y_{i}{ }^{\prime} & z_{i}{ }^{\prime}\end{array}\right)$. Let the thickness of the cross-section bed then the points in the section point set satisfy $\mathrm{M}\left(\begin{array}{lll}x_{i} & y_{i} & z_{i}\end{array}\right)$ :

$$
d \geq \frac{\left|x_{i}-x_{p}+y^{\prime}\left(y_{i}-y_{p}\right)+z^{\prime}\left(z_{i}-z_{p}\right)\right|}{\sqrt{x^{\prime 2}+1+z^{\prime 2}}}
$$

By rotating twice, the $\mathrm{X}$-axis of the section coordinate system coincides with the direction of the point cloud vector $v_{1}$. The rotation angles $\alpha$ and $\beta$ are the rotation angles of the rotation matrix base around the $\mathrm{Z}$-axis and the $\mathrm{Y}$-axis respectively, The values of rotation angles $\alpha$ and $\beta$ are as follows:

$$
\alpha=\arctan y^{\prime}, \beta=\arctan \left(z^{\prime} \sqrt{1+y^{\prime 2}}\right)
$$


After adjusting the attitude of the tunnel section, the coordinate values of the intercepted tunnel section become:

$$
\begin{aligned}
& {\left[\begin{array}{l}
X \\
Y \\
Z
\end{array}\right]=R_{\beta} R_{\alpha}\left[\begin{array}{c}
X_{i} \\
Y_{i} \\
Z_{i}
\end{array}\right]} \\
& \text { Where , } R_{\alpha}=\left[\begin{array}{ccc}
\cos \alpha & -\sin \alpha & 0 \\
\sin \alpha & \cos \alpha & 0 \\
0 & 0 & 1
\end{array}\right], R_{\beta}=\left[\begin{array}{ccc}
\cos \beta & 0 & \sin \beta \\
0 & 1 & 0 \\
-\sin \beta & 0 & \cos \beta
\end{array}\right] .
\end{aligned}
$$

\section{Limit Detection Analysis.}

\subsection{Tunnel Limit Standard Size.}

According to the type of traction, design speed, and other conditions, the tunnel limit standard size is also different. Figure 4 and Figure 5 show the standard dimensions of tunnel clearance for the electric traction section and internal combustion traction section of $160 \mathrm{~km} / \mathrm{h}$ Mixed Passenger and freight railway.

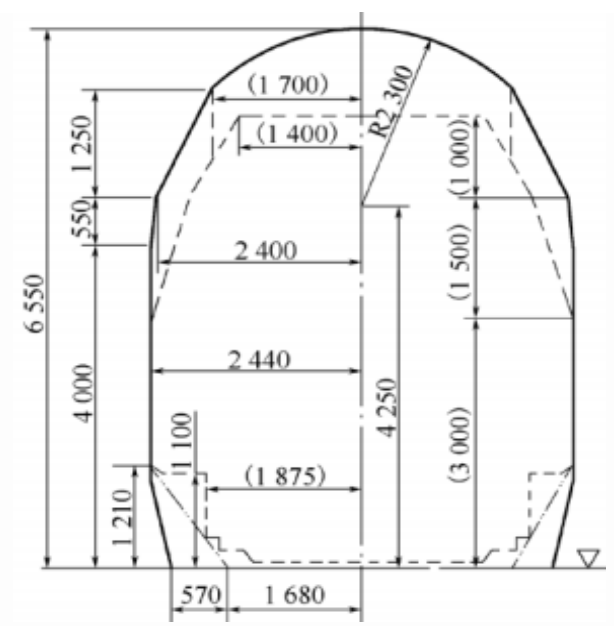

Figure 4. Tunnel clearance standard for electric traction section of passenger and freight lines (unit: $\mathrm{mm})$ 


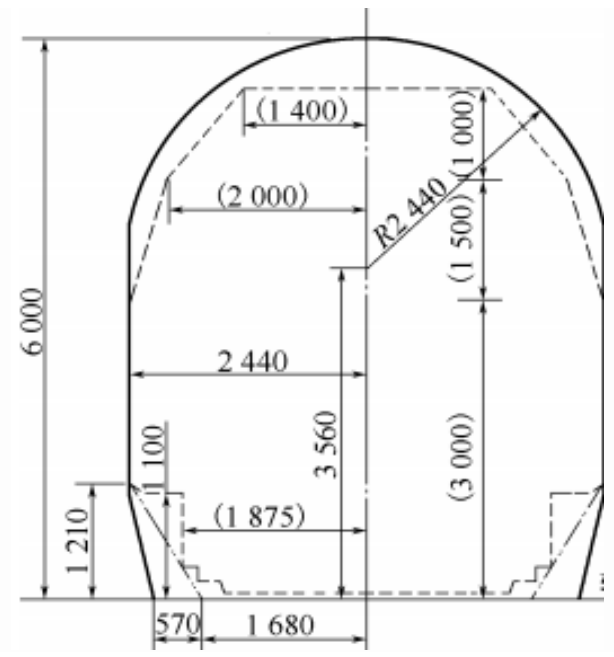

Figure 5. Tunnel clearance standard for diesel traction section of passenger and freight lines (unit: mm)

\subsection{Establishment of Tunnel Gauge Detection Coordinate System}

According to the extracted tunnel cross-section, the rail surface is regarded as the measurement datum. The tunnel gauge detection coordinate system $\left(O^{\prime}, X^{\prime}, Z^{\prime}\right)$ is a rectangular plane coordinate system with the rail surface point line of the left and right rails as the $X^{\prime}$ axis, and the plumb line where the midpoint of the vertical rail top connection line is the $Z^{\prime}$ axis. The coordinate origin $O^{\prime}$ is the midpoint of the left and right rail surface points.

\subsubsection{Track Point Extraction}

According to the definition of the above-mentioned gauge detection coordinate system, the basis of establishing the tunnel gauge detection coordinate system is to determine the rail surface points of the left and right rails. In the cross-section of the tunnel, the extraction process of track points on both sides is divided into two steps: rough extraction and fine extraction.

(1) Firstly, the point cloud of the tunnel wall on both sides of the section is filtered according to the coordinate of the central axis and the limitation of the gauge. Combined with the height limit, the point cloud of the vault is filtered, Thus, only the rail and its nearby ground point cloud are retained.

(2) According to the linear characteristics of the rail, the rail surface points on both sides of the cross-section are found, which is to screen the points with the maximum elevation of the left and right tracks in the remaining point clouds to realize the accurate extraction of rail surface points.

\subsubsection{Coordinate System Transformation}

To judge whether the section contour point intrudes into the gauge standard, It is also necessary to transform the coordinate system $\left(O^{\prime}, X^{\prime}, Y^{\prime}\right)$ of the section contour point 
into a coordinate system $\left(O^{\prime}, X^{\prime}, Y^{\prime}\right)$ unified with the tunnel clearance detection coordinate system, as shown in Figure 6.

The migration parameters between the two coordinate systems are $\Delta x$ and $\Delta y$. When the tunnel is located in a curve section, the angle between axis $X$ and $X^{\prime}$ axis will be $\theta$. According to the rotation translation transformation formula of the rectangular coordinate system, the points in two coordinate systems satisfy the following requirements:

$$
\left[\begin{array}{c}
x \\
z
\end{array}\right]=\left[\begin{array}{cc}
\cos \theta & -\sin \theta \\
\sin \theta & \cos \theta
\end{array}\right]\left[\frac{x^{\prime}}{y^{\prime}}\right]+\left[\frac{\Delta x^{\prime}}{\Delta y^{\prime}}\right]
$$

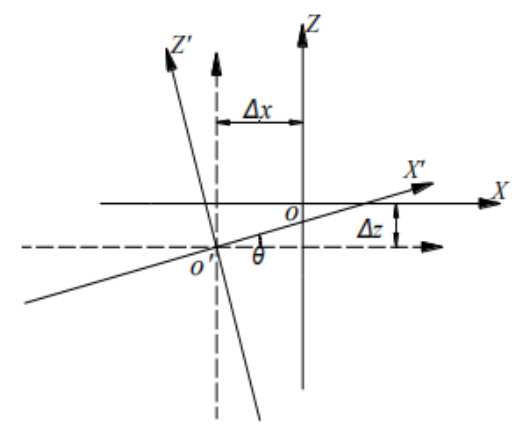

Figure 6. The coordinate system conversion schematic.

\subsection{Limit Violation Discrimination}

The point cloud collected by a 3D laser scanner is a series of scanning contour points. Geometrically, the standard size of railway tunnel gauge is a polygon. Therefore, the 3D collision problem in gauge detection can be transformed into the position relationship between points and polygons on the plane. In this paper, the improved ray algorithm is used to determine whether the contour points of the section scan are located in the boundary polygon. As shown in Figure 7, this paper further optimizes it so that the method can further give the size of the intrusion limit.

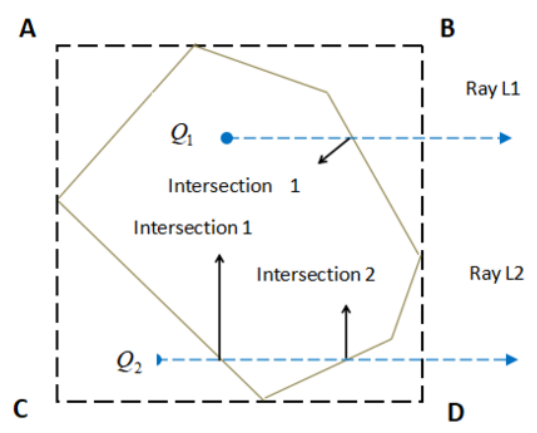

Figure 7. improved ray method 
(1) First, we judge whether the point $\mathrm{Q}\left(x^{\prime}, z^{\prime}\right)$ is in the polygon bounding rectangle. By traveling to get the polygon area of $x_{\max }, x_{\min }, z_{\max }, z_{\min }$. For the point, if $x<x_{\max }$, $x^{\prime}>x_{\text {min }}, z^{\prime}<z_{\text {max }}, z^{\prime}>z_{\text {min }}$ conditions are established at the same time, then the next judgment, whereas the point is located outside the polygon.

(2) Taking this point as the starting point, a ray is drawn to infinity, and the number of intersections between this ray and polygon is calculated. If it is odd, the point is inside the polygon, if it is even, it is outside the polygon.

(3) If point $Q$ is located in the interior of the polygon, it can be judged as the limit point. Traversing the distance di from each edge of the bounding polygon to find the minimum distance is the limit value.

\section{Actual Engineering Verification}

\subsection{Project Overview and Data Collection}

The experimental data were collected from the tunnel site of the Bao Shen railway, the area is undulating and gully developed. The geological conditions of the tunnel are complex. With the increasing traffic density, tunnel diseases are becoming increasingly prominent. It is urgent to investigate the tunnel diseases and evaluate the tunnel operation status, measurement site is shown in Figure 8,

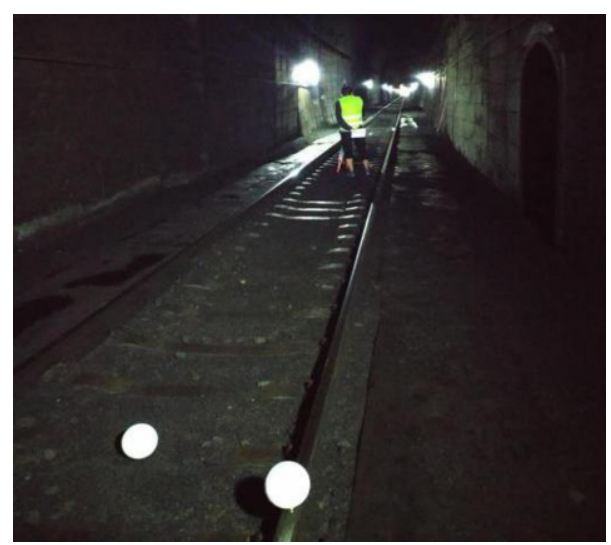

Figure 8. The engineering site.

$Z+F$ imager 5010c 3D laser scanner was used. According to the chord length formula (5), we can estimate the distance $L$ between adjacent points at different distances $\mathrm{R}$ in the scanning area.

$$
L=2 R \sin \left(\frac{\delta}{2}\right)
$$

The field operation uses the scheme of setting up "I-shaped" stations in the centerline of the road along the route, and the selected station spacing is $25 \mathrm{~m}$. As can be 
seen from the calculation of the formula (8), it is guaranteed that the point cloud interval within the scan area is less than $0.3 \mathrm{~mm}$. The collected experimental data sets are described in Table 1.

Table 1. Experimental data sets

\begin{tabular}{cccc}
\hline Test section location & Curve radius / M & Length / M & $\begin{array}{c}\text { Number of stations / } \\
\text { stations }\end{array}$ \\
\hline Tunnel entrance & straight line & 200 & 8 \\
\hline
\end{tabular}

\subsection{Point Cloud Data Processing and Accuracy Analysis}

The point cloud of tunnel entrance section after splicing, denoising, and other pretreatment is shown in Figure 9. Based on fitting the central axis with quadratic polynomial, the continuous extraction of the tunnel section is realized. In this paper, the interval between sections is taken as $1 \mathrm{~m}$. Considering the requirements of point cloud density and tunnel clearance detection accuracy, the section thickness threshold D is taken as $1 \mathrm{~cm}$. The results of tunnel section extraction are shown in Figure 10.

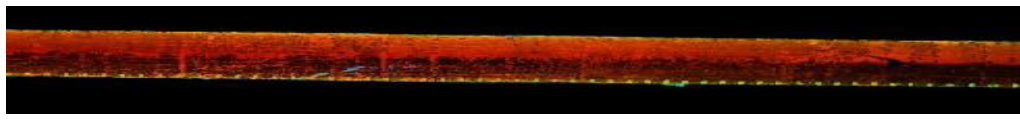

Figure 9. The tunnel entrance section points down the clouds.

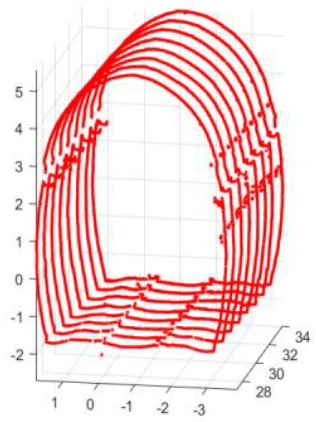

Figure 10. Continuous extraction of tunnel sections

Through the extraction of rail surface points, the gauge detection coordinate system is established. To analyze the feasibility of the section extraction method and the accuracy of the gauge detection coordinate system, six tunnel sections were selected. Calculate the distance $\mathrm{L}$ between the two rails and take the gauge distance value measured by the gauge as the true value. The gauge refers to the shortest distance between two rails $16 \mathrm{~mm}$ below the rail top surface from the inside of the rail. Compare the sum of gauge value, a railhead width value, and the $\mathrm{L}$ value of each section. The error of the gauge detection coordinate system is $6 \mathrm{~mm}$ after averaging the error of measurement results. It can be seen that the results are in line with the engineering practice. It can meet the requirements of railway tunnel clearance detection accuracy. 


\subsection{Test Results}

In this experiment, according to the standard size of tunnel gauge in the electric traction section of passenger and freight railway with $\mathrm{v}=160 \mathrm{~km} / \mathrm{h}$, the above method is used to program the tunnel for limit violation detection. The experimental results show that: as shown in Figure 11, a cross-section violation is detected in the straight-line section of the exit. The section is located $4 \mathrm{~m}$ away from the exit. It is found that the cable trough and the bottom of the track on the right side of the tunnel are both intruded. Enlarging the local map of the right wall's intrusion position, as shown in Figure 12, can intuitively represent the situation of tunnel clearance invasion.52 points were detected on the cross-section of the tunnel. And the maximum limit is $9.2 \mathrm{~mm}$.as is shown in Figure 13.

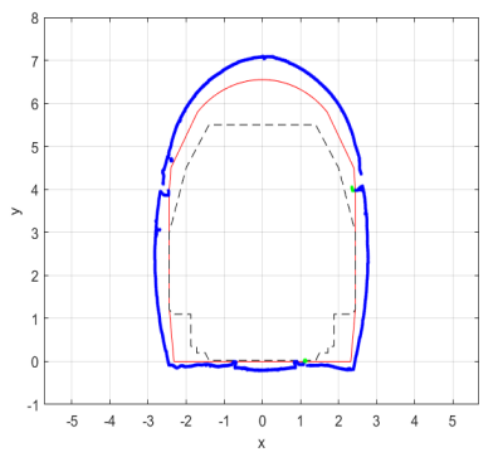

Figure 11. The entrance section is inaccessible to the tunnel section at the limit.

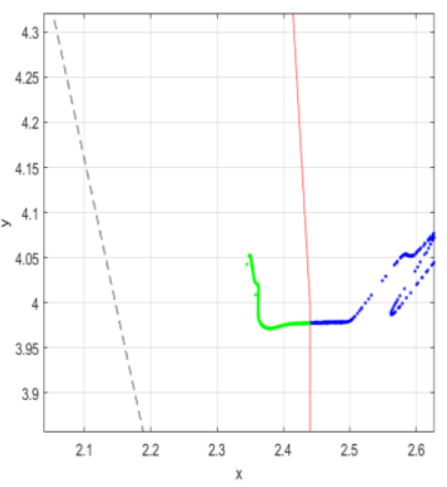

(a)Limit point of right wall invasion

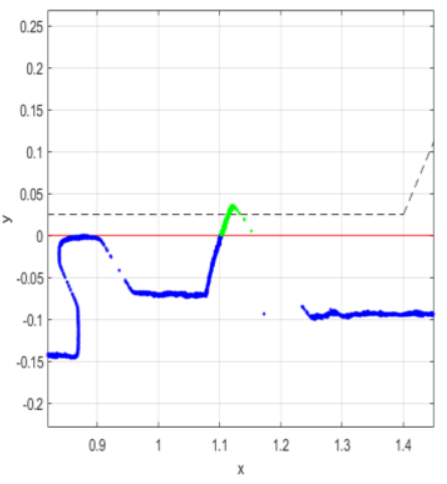

(b) The bottom arch point.

Figure 12.the enlarged diagram of intrusion position .

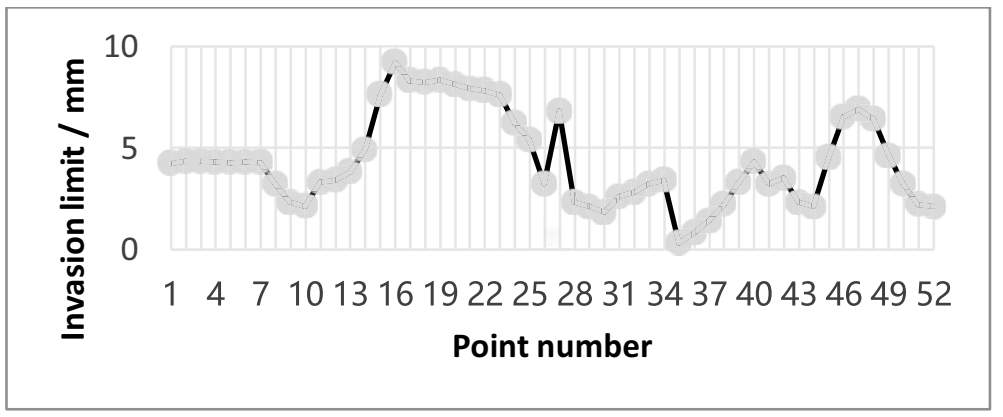

Figure 13. The invasion limit distribution diagram. 


\section{Conclusion}

Through the method of dimensionality reduction projection transformation, the differential step length method is used to obtain the point cloud boundary point set, and finally the central axis of the tunnel space is obtained. After the point cloud is rotated and transformed according to the central axis, the tunnel cross section can be extracted at any position of the tunnel at equal intervals. A detection method based on rail modeling is proposed, and a tunnel gauge detection coordinate system based on the rail top surface is constructed. The problem of three-dimensional middle limit detection is transformed into the position relationship between points and polygons on the plane. Different types of clearance frames are introduced to calculate the limit intrusion with the tunnel section, the improved ray algorithm is used to detect the clearance automatically. Experiments show that the accuracy of this method can meet the engineering applications, and it can also accurately identify the position of the intrusion limit point, and give the size of the intrusion limit and the cross-sectional view of the intrusion limit, which has a certain practicability and feasibility in tunnel boundary detection.

\section{References}

[1] 2014 T G. Railway Technical Management Regulations .

[2] Puente I, Akinci B, González-Jorge H, et al. A semi-automated method for extracting vertical clearance and cross sections in tunnels using mobile LiDAR data. Tunnelling and Underground Space Technology, 2016, 59: 48-54.

[3] Chi TN, Alexandr G. Hyperstatic reaction method for calculations of tunnels with horseshoe-shaped cross-section under the impact of earthquakes. Earthquake Engineering and Engineering Vibration, 2020, 19(1): 179-188.

[4] Hu QW, Chen ZY, Wu S. Fast automatic detection method of railway building boundary based on moving binocular vision. Journal of the China Railway Society, 2012, 34(1): 65-71.

[5] Zhou Y, Wang S, Mei X, et al. Railway tunnel clearance inspection method based on 3D point cloud from mobile laser scanning. Sensors, 2017, 17(9): 2055.

[6] Li P, Tao JQ, Liang XF, et al. An improved detection method for railway out-of-gauge freight transportation simulation limits. Journal of Central South University: Natural Science Edition, 2017, 48(2): 547-552.

[7] Du LM, Zhong RF, Sun HL, et al. Tunnel cross section extraction and deformation analysis based on mobile laser scanning technology. 2018.

[8] Kang Z, Zhang L, Tuo L, et al. Continuous extraction of subway tunnel cross sections based on terrestrial point clouds[J]. Remote Sensing, 2014, 6(1): 857-879.

[9] Ma HY. The application of three-dimensional laser scanner in tunnel construction. Highway Engineering, 2018, 43(03): 96-100.

[10] Wang M, Feng J, Xie ZH. Using 3D laser scanner to measure subway tunnel boundary. Bulletin of Surveying and Mapping, 2014 (12): 78-81.

[11] Li J, Wan Y, Gao X. A new approach for subway tunnel deformation monitoring: high-resolution terrestrial laser scanning. ISPAr, 2012, 39: 223-228.

[12] Han F. Research on Existing Railway Status Detection and Evaluation Technology Based on Point Cloud Information. Southwest Jiaotong University, 2015.

[13] Nuttens T, De Wulf A, Bral L, et al. High resolution terrestrial laser scanning for tunnel deformation measurements//FIG Congress. 2010, 2010.

[14] Han S, Cho H, Kim S, et al. Automated and efficient method for extraction of tunnel cross sections using terrestrial laser scanned data. Journal of computing in civil engineering, 2013, 27(3): 274-281.

[15] Torrey, Kang ZZ, Xie YC, et al. Research on continuous interception method of subway tunnel section using 3D point cloud data. Journal of Wuhan University Information Science Edition, 2013, 38(2): 171-175,185.

[16] Zhou SM. Tunnel boundary detection method based on mobile laser scanning 3D point cloud. Sichuan Building Materials, 2018, 44(1): 174-180. 
[17] Yang YJ, Duan MY. An improved algorithm for judging the relationship between a point and a designated polygon area. Computer Knowledge and Technology: Academic Exchange, 2014 (8): 5362-5364. 